\title{
Editorial
}

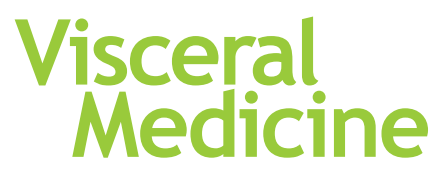

\section{Malnutrition and Intestinal Failure}

\author{
Georg Lamprecht ${ }^{\mathrm{a}}$ Irina Blumenstein ${ }^{\mathrm{b}}$

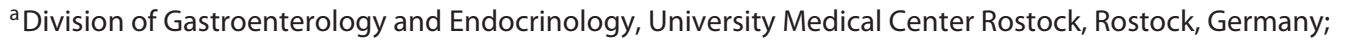 \\ ${ }^{b}$ Medizinische Klinik 1 - Gastroenterologie und Hepatologie, Pneumologie und Allergologie, Endokrinologie und \\ Diabetologie sowie Ernährungsmedizin, Universitätsklinikum Frankfurt, Frankfurt/M., Germany
}

Adequate nutrition is a fundamental physiological function comparable to electrolyte homeostasis and coagulation but with a different time frame both in its deterioration and its (re)compensation. Nutritional status may be diminished as a result of reduced intake and/or increased demand and "catabolic drive" related to ageing and/or concomitant disease. Intestinal failure is the relatively rare situation with an opposite pathophysiology, in which malnutrition arises from diminished absorptive capacity. While malnutrition has long been recognized as a complicating factor for the outcome of many diseases, obesity has only recently emerged as a disease state, which is invasively being treated using bariatric surgery. Finally, sarcopenia has emerged as a specific pathophysiological condition which often accompanies or follows malnutrition, and which carries an even more serious risk.
Despite this common understanding of malnutrition in the field of visceral medicine, the approach to the various clinical situations is by far not uniform or standardized due to lack of high-quality data and more so due to lack of awareness of the existing data. In the current issue of Visceral Medicine, experts in the field review important aspects of malnutrition and intestinal failure starting from definitions and diagnostic criteria to prophylaxis/ prehabilitation and to specific situations such as liver disease and malnutrition in the context of obesity and bariatric surgery. Modern surgical and medical approaches to intestinal failure with its opposite pathophysiology are covered as well. In addition, the interdisciplinary discussion clearly points out the importance of an interprofessional and transsectoral approach as well as the medicoeconomic hurdles that have to be overcome to make it happen.

\section{KARGER}

(C) 2019 S. Karger AG, Basel
Prof. Dr. Georg Lamprecht

Division of Gastroenterology and Endocrinology

University Medical Center Rostock

Ernst-Heydemann-Strasse 6, DE-18057 Rostock (Germany)

E-Mail georg.lamprecht@ med.uni-rostock.de 